\title{
Knowledge for Courseware Engineering: A Framework for Inductive Knowledge Acquisition Based Upon Evaluation of Adaptable Courseware Products
}

\author{
Al-Noor Ladhani and Italo De Diana
}

University of Twente

\begin{abstract}
Authors or adaptors of courseware products preferably should receive support in the process of development and adaptation of courseware products. A predictive agent is defined as a system that is able to predict the expected effectiveness of various composable products from current product attributes. The described research addresses the questions of how to acquire the necessary knowledge for a predictive agent, how to organize this knowledge, and how to link it with methods and tools for courseware authoring and adaptation. We propose to use a methodology, derived from the field of machine learning, and present a framework for applying inductive knowledge acquisition based upon the empirical evaluation of adaptable courseware products.
\end{abstract}

\section{INTRODUCTION}

Traditionally, tutorial courseware products are developed in a fixed form. The users of such products, teachers and learners, are not able to alter product attributes. Products may have been fixed for a number of practical reasons, such as to be distributable as compiled products, or for pedagogical reasons, such as the assumption that users are not the ones to make decisions concerning product attributes. Yet, a growing need is felt for adaptable products that can be modified by users to serve their own needs and preferred learning style (De Diana \& de Vries, 1990; Moonen, 1989).

A courseware product is called adaptable if the way it interacts with learners can be changed by means of parameter settings within a range of already available options. Several types of properties can be adjustable. Usual product adjustments 
concern hardware configurations like a video adapter or an input device. At a more sophisticated level, ergonomical configurations, as, for example, font size, font colors, the size of the windows, and the number of windows, are adjustable. One step further still, one could think of adjustable instructional configurations like microand macrostrategies. According to Merrill (1983), microstrategies are concerned with details of individual presentations to the learner, including their characteristics, interrelationships, and sequence. An example of a microstrategy for learning to use a concept is to first present a definition, followed by a number of examples. Next, let the learner practice by classifying unseen examples and nonexamples, and finally, let the learner perform by correctly classifying unseen examples and nonexamples. Macrostrategies, according to Merrill, are concerned with the selection, sequencing, and organization of the subject matter topics that are to be presented. Ladhani and De Diana (in press) describe a number of dimensions on which courseware products may be adaptable.

Instructional transactional shells, as defined by Li and Merrill (1990), can also be conceived of as adaptable courseware products. Such shells hold a large number of instructional parameters which enable computerized instruction to be customized for a given learner population, learning task, and environmental situation. The shells contain functions for easily setting instructional parameters but do not yet offer much help to a nonexpert in choosing appropriate values for these parameters. Appropriate values in this context are considered to be parameter values that promote the realization of instructional, learning, and organizational goals.

De Diana and de Vries (1990) have proposed to expand the traditional courseware life cycle, consisting of the stages specify, design, develop, use, and evaluate, with two new stages - namely, adapt before use and adapt after use. An adaptable courseware product is specified, designed, and developed in the "production" phase by a first author, which is a generic term for professional courseware producers. In the use phase, the product is adapted, used, evaluated, and adapted after use. Adaptation and evaluation is performed by a second author or an adaptation agent, most likely a teacher. Learners can also act as adaptation agents if they are able to adapt the product to their own needs or tastes. Yet another adaptation agent might be the program itself; in this case one could refer to the agent as belonging to the family of intelligent tutoring systems (Wenger, 1987).

In order to make product adaptation a feasible and efficient process for second authors, mainly teachers and learners, it is necessary to support them. Support can be given in two ways:

1. By offering software tools and courseware "building blocks" that are especially suited for adaptational purposes. De Diana and de Vries (1990) have described a "Use Environmen" for adapting, using, and evaluating adaptable courseware products. The use environment consists of a number of integrated software tools to support courseware adaptation, in addition to a library with adjustable courseware building blocks.

2. Second authors can be given explicit knowledge to guide the decision process involved in making product adjustments. In order to be really effective for a second author, this explicit knowledge should be represented in an executable form, instead of merely being described in a theory or model. Two categories of support systems embodying executable representations of knowledge are expert systems and on-line help systems.

The first form of support is important for product adaptation to become a feasible and effective process; the second form is essential if a second author, who can 
be considered a nonexpert, is to carry out the adaptation process. The second form of support, though, is more difficult to accomplish, basically due to the complexity of explicating knowledge that can be used to support a second author. The activity of extracting knowledge and representing it in a formalism that enables reasoning with this knowledge is called knowledge acquisition.

Figure 1 summarizes the entities and their interrelationships that will be elaborated in this article. The entities shown are involved in supporting the adjustment of adaptable courseware products with explicit knowledge. The way an adaptable courseware product interacts with learners can be modified by changing parameter settings within an already available range of options. The second author is the adaptation agent involved in adapting the courseware product to his personal needs or tastes. In order to support the second author, it is necessary to provide him with explicit and executable knowledge about options for product adaptation. We will first point out that knowledge that enables a second author to reason about the effects of adaptation on learning outcomes is likely to have substantial impact on the adaptation process. We will use the term product knowledge to indicate this type of knowledge. Next, we will introduce a predictive agent, embodying executable product knowledge. A predictive agent is capable of supporting a second author by predicting effects of adjustments on learning outcomes. A hard problem in building a predictive agent is acquiring the knowledge instances that enable reasoning, necessary to predict adaptation effects. In this article we suggest applying an inductive knowledge acquisition method to acquire the necessary knowledge. This method is based upon the acquisition of product knowledge during the lifecycle of adaptable courseware products.

\section{ADAPTATION KNOWLEDGE}

While adapting a courseware product, the second author is involved in a complex problem solving task. In this section, we will classify the knowledge necessary to

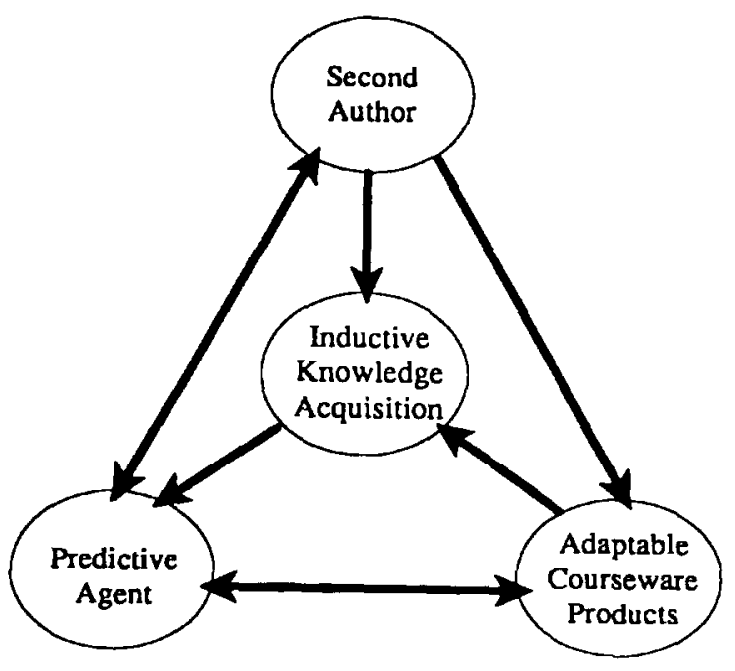

Figure 1. Entities involved in supporting the adjustment of adaptable products with explicit knowledge. 
solve the adaptation problem into product, process, and tool knowledge. Next, we will point out that these three types of knowledge can either consist of synchronic or diachronic types of relations or both.

\section{Product, Process, and Tool Knowledge}

Product knowledge refers to knowledge that relates to product attributes. The term product attribute is used here in a wide sense, encompassing attributes of product users and the intended and factual effects of product use. Process knowledgc refers to knowledge instrumental in constructing a product, and the term tool knowledge is used to express knowledge about tools that can be used to construct a product. Suppose that you are writing a conference paper. Product knowledge, in this case, refers to knowledge about attributes of the paper itself (e.g., concerning its contents, its aims, the target audience, and the name of the author). Process knowledge refers to knowledge about methods usable in the writing process. One could, for example, start by writing an outline, followed by references, and then expand the outline step by step with content materials. Another method would be to report first about experiments performed and the experimental outcomes, then about the theoretical framework, and finally to add abstract, conclusions, and references to the paper. Tool knowledge refers to knowledge about how to use tools that are involved in writing the paper. In the example given, the tools usually will consist of a computer and a word processor package. In effect, the tool knowledge would mainly concern knowledge about the command options of the word processor.

\section{Synchronic and Diachronic Knowledge}

A second classification scheme for knowledge types involved in adjusting adaptable products differentiates between knowledge consisting of synchronic relations and knowledge consisting of diachronic relations. Their difference is situated in the question of whether or not time is encapsulated in the relations. The terms synchronic and diachronic are used by Holland, Holyoak, Nisbett, and Thagard (1986) to distinguish between synchronic rules that are atemporal in a subjective sense and diachronic rules that specify the manner in which the environment is expected to change over time, either autonomously or in response to outwarddirected actions of some system involved. Expressing the distinction from a different point of view, synchronic relations do not alter the state of the world, while diachronic relations carry out operations which will change the state of the world. Examples of synchronic relations are "A chair has four legs and a back" and "If an object has four legs and a back then the object is a chair." Examples of diachronic relations are "If a leg of a chair breaks then the chair will fall down" and "If an object heavier then 300 pounds is put on the chair then one or more legs of the chair will break."

\section{A Need for Explicating Synchronic Product Knowledge}

In our research we limit ourselves to the study of synchronic product knowledge in relationship to the adaptation of courseware products. Our expectation is that while adjusting an adaptable courseware product, the second author will especially have difficulties in estimating the effects of particular adjustments on learning outcomes of a group of learners with specific learner attributes. According to Zachary (1988), people tend to have substantial difficulty in making good decisions if many com- 
peting attributes or objectives have to be heuristically combined using subjective argumentation. Zachary calls the remaining residues of mental tasks that defy precise descriptions "judgments." Explicating judgments in product knowledge is a hard task.

Both process and tool knowledge can be made more easily explicit than product knowledge. In general, more is known about likely approaches to go about adapting a product and about the use of adaptation tools than about the effects of adaptations on learning outcomes. So, the need to explicate product knowledge is considered to be more compelling than the need to explicate both other forms of knowledge.

Limiting our research to synchronic knowledge has been based upon the assumption that product knowledge consisting of synchronic relations only will serve to describe various relevant product adaptations and corresponding attribute values. Explicating and representing diachronic knowledge is far more difficult then acquiring executable synchronic knowledge (Muggleton, 1990). We intend to study the use of diachronic product knowledge in a later stage of our research.

\section{A Basic Assumption Concerning Product Knowledge}

Product attributes can be functionally grouped, such as with ergonomical attributes and instructional attributes. By means of the adaptation process, product attributes receive attribute values. By functionally grouping the attributes, the received values can be represented in vectors of values or "patterns." We assume the existence of actual and clear relationships between patterns of product attribute values and learning outcomes of a group of learners with specific learner attributes. Knowledge acquisition in a world without any regularities is useless activity. We assume that our world does contain regularities; our goal is to define ways to explicate these regularities in an efficient and effective way and to use the explicated relations to support the second author.

We will discuss product knowledge from now on only on the basis of synchronic relations between product attributes, learner attributes, and expected learning outcomes.

\section{Representing Product Knowledge}

Woods and Roth (1988) stated that visualization can be a powerful aid to comprehension and conceptualization of information. Product knowledge can be represented in a descriptive, textual way as, for example, in rules. We propose to use a representation formalism for product knowledge that is visual in nature and usable for the process of product knowledge acquisition. We refer to this visual formalism as the hyperspace representation. A hyperspace is a $n$-dimensional space, separable by $n-1$ dimensional planes or hyperplanes. The planes enclose regions of meaning. Each region in the hyperspace can identify a set of specific learning outcomes. The axes that dimension the hyperspace represent product attributes that are adjustable by parameter assignment. Valued product attributes are specific points on the axes of the hyperspace. The intersection of specific values on the axes of the hyperspace locate a region identifying a learning outcome that is expected according to the product attribute set. The hyperspace can consist of dimensions that not only represent product attributes but that also represent learner attributes.

Consider the following simple example of a two-dimensional hyperspace with one adjustable product attribute, one learner attribute, and a two-valued learning outcome. The adjustable product attribute is whether the courseware product 
should or should not use color in presenting information to the learner. The learner attribute is whether the learner is color-blind or not. For this toy example, the learning outcome is simply indicated as a good or bad result. Figure 2) shows a hyperspace and the hyperplanes for this example. In this case, the hyperspace is a two-dimensional space, which is a plane. The hyperplanes are one-dimensional lines. The hyperspace representation shown in Figure 2 is not the only solution for the color-blind/use color classification. A limited translation or rotation of the hyperplanes may still represent a correct classification. It should be noted that hyperspaces containing more than three dimensions can not be visualized directly, but can be virtually represented by generalizing from principles we know from one-, two-, and three-dimensional spaces to higher dimensional spaces. A visual inspection of higher dimensional spaces is possible by projecting higher order spaces from different angles on three- or two-dimensional spaces.

In the hyperspace representation, a knowledge acquisition problem consists of (a) explicating the dimensions of product attributes and learner attributes, (b) explicating valid parameter values for each of these dimensions, (c) explicating possible learning outcomes, and (d) finding hyperplanes that separate the hyperspace into regions containing comparable expected learning outcomes.

We assume that we already have an adaptable product, a classification of learner attributes, and a classification of learning outcomes. The product knowledge we intend to represent only contains relations between product attributes, learner attributes, and the expected effect of these attributes on learning outcomes. In other words, while adapting a product, the dimensions of the hyperspace, valid parameter values for each of these dimensions, and valid values for learning outcomes are considered to be known. The knowledge acquisition problem thus consists of locating appropriate hyperplanes in hyperspace and providing the regions enclosed by the hyperplanes with appropriate learning outcome values.

An important term for discussing the hyperspace representation is the word granularity. The granularity of a hyperspace is related to the size of its regions. A hyperspace can contain regions of different size. If the granularity of a hyperspace is high, then the space consists of a small number of regions that are mainly significant in size. In case of low granularity, the hyperspace contains a considerable number of small regions. We refer to a hyperspace with high granularity as a sparse hyperspace, while the term dense hyperspace is used to indicate a hyperspace with low granularity.

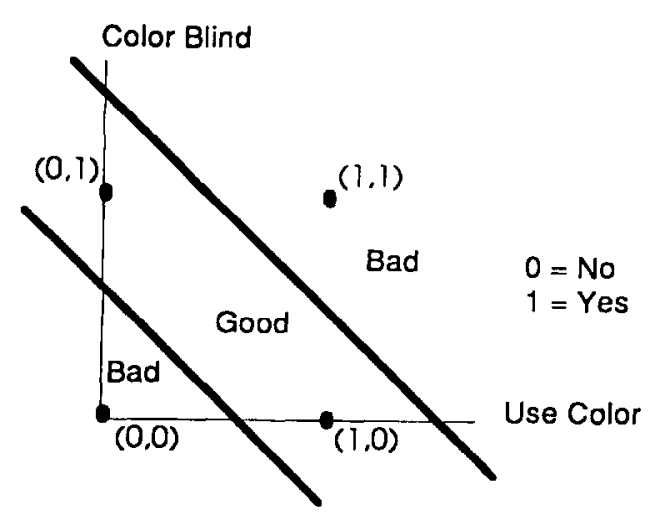

Figure 2. An example of representing product knowledge as a hyperspace with hyperplanes and regions. 


\section{THE PREDICTIVE AGENT}

The intended support system for the second author has been termed predictive agent. A conceptual architecture for a predictive agent is shown in Figure 3. A predictive agent consists of a hyperspace representation-based knowledge base, an inference engine, human-computer interface, and an access facility for inspecting and editing the knowledge base. Through the human-computer interface, the second author provides the predictive agent with intended adjustments of which a prediction of expected learning outcomes is desired. The predictive agent uses its inference engine and its knowledge base with product knowledge to reason about the effects of intended adjustments. The result, a prediction, is presented to the second author through the human-computer interface. The access facility to the knowledge base can be used by a human or by an automated system.

The predictive agent should at least satisfy four criteria. First, we consider the brittleness of the agent. Knowledge-based systems are called brittle if they are only usable for narrow problem domains and, in case of slight changes in the domain, have to be revised considerably, or even reconstructed. According to Holland (1986), ultimately brittleness can only be avoided by using reflective, self-learning knowledge-based systems. He proposes to use induction as the basis for automatic improvement of a knowledge base, using feedback from the environment in order to increase the performance of the knowledge system. Holland assigns a "strength" to each rule in a rule-based system. The strength is modified on the basis of the rule's overall usefulness as the knowledge system gains experience. The difficult task of generating plausible new rules is carried out by a so-called genetic algorithm. Reducing brittleness also requires avoiding situations in which a predictive agent, although provided with valid input by a second author, does not return an output. In terms of the hyperspace representation, reducing brittleness requires hyperplanes that are not fixed but that can be translated and rotated according to

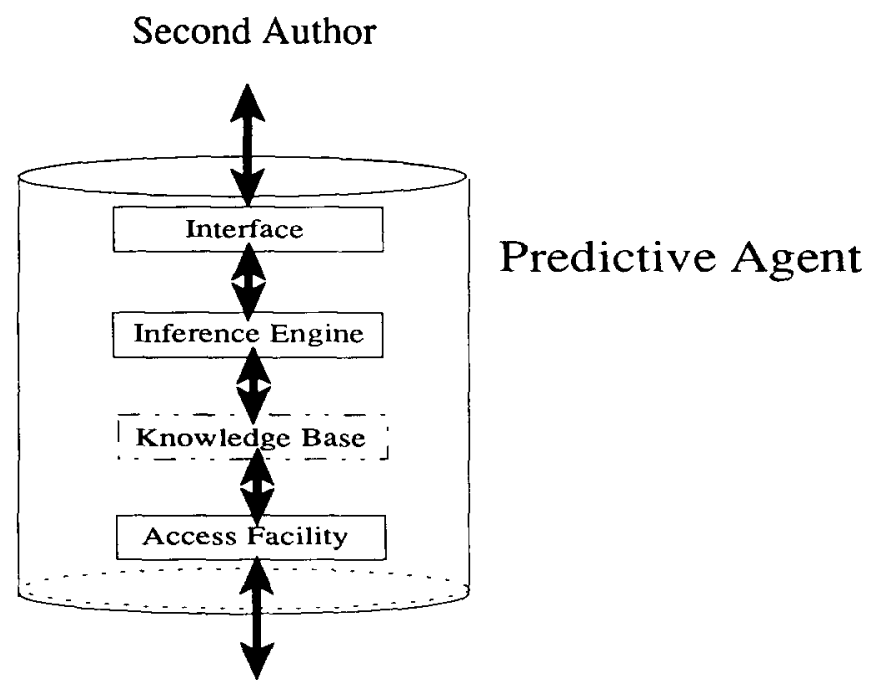

Knowledge Editor

Figure 3. A conceptual architecture of a predictive agent. 
feedback from the environment in response to the performance of the knowledgebased system.

Second, the behavior of the predictive agent should demonstrate an appropriate degree of granularity in order to be of use to the second author. In terms of the hyperspace representation, the knowledge acquisition task consists of locating hyperplanes in the hyperspace enclosing regions offering comparable learning outcomes. The process of knowledge acquisition has to ensure an adequate degree of granularity. In case of high granularity, the hyperspace will be sparse, only containing a few large regions. If a predictive agent is to work based on a sparse hyperspace then it tends to generalize its advice. On the other hand, very fine-grained processes of knowledge acquisition may result in a dense hyperspace, containing a significant number of small regions. If a predictive agent is to work on the basis of a dense hyperspace then it tends to become unnecessarily specific. Clearly, more generalizations should have been made while the knowledge was acquired.

In the third place, the predictions made should be reliable. A predictive agent may work from appropriate granularity, but if the hyperspace does not represent reliable relations then the agent is of no practical use to the second author.

In the fourth place, the predictive agent should be machine plausible, meaning that it can be executed on a machine with limited storage and processing capacities, and that the results are calculated within an acceptable time limit.

\section{THE KNOWLEDGE-ACQUISITION PROBLEM}

A major difficulty in constructing a predictive agent is finding appropriate hyperplanes that separate a hyperspace into regions of expected learning outcomes. Three sources of knowledge can be considered for the acquisition of product knowledge - namely, human experts, instructional design theories and models, and existing courseware products.

\section{Knowledge Acquisition From Human Experts}

The process of explicating knowledge from a human expert is usually referred to as knowledge elicitation. To structure the complicated elicitation process, many techniques and tools have been developed in the last decade; Boose (1989) gives a survey. Still, the process of elicitation is often complex and time consuming, and the outcomes often do not reflect initial expectations. Three additional problems can be identified in the elicitation of product knowledge. The first problem lies in the task of finding an appropriate expert. According to Salter (1988), the experts must be genuine experts; they should be verbally expressive and relatively introspective, they must believe in the value of the overall enterprise, and they must participate out of interest. But even when succeeding in the mission of finding genuine experts, product knowledge typically consists of a significant amount of judgments. A second problem is caused by the situation that, although the human expert is able to make certain inferences involving product knowledge, he or she is often not really able to explain in detail why and how they are made. After the process of elicitation and representing the results in a knowledge base, the knowledge is often only used to draw inferences, and the knowledge base itself is not subject to any changes. The third problem is that reducing brittleness, finding appropriate degree of granularity, and improving reliability often require extensive debugging, extend- 
ing, and refining of the knowledge base. The reason for not extensively altering the elicited knowledge lies in the hard task of modifying this knowledge. The modification is usually done manually by knowledge engineers, and often requires additional elicitation of expert knowledge. This is a time consuming, and therefore expensive, activity for both the knowledge engineer and the human expert.

\title{
Knowledge Acquisition From Instructional Design Theories and Models
}

Another strategy is to try to deduce product knowledge from existing instructional design theories and models. Merrill and $\mathrm{Li}(1989)$ have constructed an instructional design expert system which, among other things, provides advice for selecting and adapting instructional modules that performed a typical instructional interaction between a learner and subject matter materials. These software modules are called transactions. The knowledge in the instructional design expert system is based on the component design theory (Merrill, 1987) and the component display theory (Merrill, 1983), which are some of the most practical instructional design theories.

An important conclusion from this project was that the current state of art of instructional design theories and models still lacks a comprehensiveness and completeness which would enable us to draw inferences to deduce specific product knowledge. As Merrill, Li, and Jones (1990) put it:

\begin{abstract}
One major approach is to improve the efficiency by which current instructional design theory and methods are applied, by developing expert systems for advice and guidance of designers. This is a conservative, knowledge engineering approach which focuses on representing existing expertise about instructional design in an expert system. The drawback of this approach is the state of knowledge about instructional design, which we have stated is inadequate for the task to which it is put. (p. 13)
\end{abstract}

\section{Knowledge Acquisition From Existing Courseware Products}

Many courseware products have been developed throughout the years. For the purpose of knowledge acquisition, one could think of using a subset of these products and, if available, the empirical evaluation of these products. The knowledge acquisition effort would consist of explicating product knowledge contained in existing products and trying to integrate the elicited knowledge about specific products into a more comprehensive body of generic product knowledge. Example-based knowledge acquisition approaches have to struggle with the hard problem of making valid generalizations from instances (Muggleton, 1990).

Another drawback of this example-based approach is that existing courseware products vary enormously, and therefore comparing different courseware products is a hard task. It is difficult to automate this knowledge acquisition task working from a range of different products.

\section{Inductive Knowledge Acquisition From Empirical Evaluation of Adaptable Products}

The knowledge acquisition problem we presently address is not solvable by using a traditional approach. We do not presume that all required knowledge could be acquired by using standard knowledge acquisition techniques such as explicating human-expert knowledge or (re)organizing knowledge found in available literature and products. In fact, we are involved in a knowledge generation pursuit. We believe that standard knowledge acquisition techniques can be used to compose an incomplete initial knowledge base, but that additional knowledge will have to be 
acquired by using an inductive acquisition of product knowledge from empirical evaluation of adaptable products. In our opinion, ultimately the only way to create an effective predictive agent is by implementing this agent as a reflective, selflearning knowledge-based system.

In the next section, a framework for inductive knowledge acquisition based upon empirical product evaluation is presented. This framework is based on the conception that within the life cycle of an adaptable product (De Diana \& de Vries, 1990) it is possible to gather product knowledge from evaluation results obtained from systematically experimenting with variations in product attributes. The methodology for the knowledge acquisition process (i.e., to ensure the systematic gathering of knowledge) is based upon the "empirical cycle," made up of the states "perceive," "generate expectation," "test expectation," "add results to knowledge base," and "generate new expectations" (Holland et al., 1986).

\section{A FRAMEWORK FOR ACQUISITION OF PRODUCT KNOWLEDGE}

The methodology for inductive knowledge acquisition is described in a conceptual framework. The framework consists of two loops, an inner and an outer one. The framework is traversed in the steps (a) perceive, (b) generate expectations, (c) test expectations, (d) add results to knowledge base, and (e) generate new expectations. The framework is illustrated in Figure 4.

In the inner loop, the methodology serves to create a new knowledge base, or to refine or expand an existing one on the basis of a given set of (additional) product adjustments-factual learning outcomes pairs. The inner loop consists of the steps:

1. Using the predictive agent to make learning outcome predictions based upon the given product adjustments;

2. Determining the discrepancies between predicted outcomes and factual outcomes; and

3. Using this result to update the knowledge base.

This process continues until the discrepancies between predicted outcomes and factual outcomes is acceptably small.

In the outer loop, the methodology serves to refine or expand an existing knowledge base on the basis of a self-induced set of product adjustments-factual learning outcomes pairs. The steps in the loop that result from the methodology consist of:

1. Inspecting the knowledge base of a predictive agent and generating product adjustments from the contents of the knowledge base that are expected to realize substantial refinement or expansion of the knowledge base;

2. Using the adapted product to determine factual learning outcomes resulting from the generated adjustments; and

3. Using the inner loop to refine or expand the knowledge base on basis of the generated adjustment-factual outcome pairs.

This process continues until a knowledge base is formed that can effectively be used to support a second author. The outer loop can be seen as a low frequency loop with an encapsulated high frequency inner loop. 


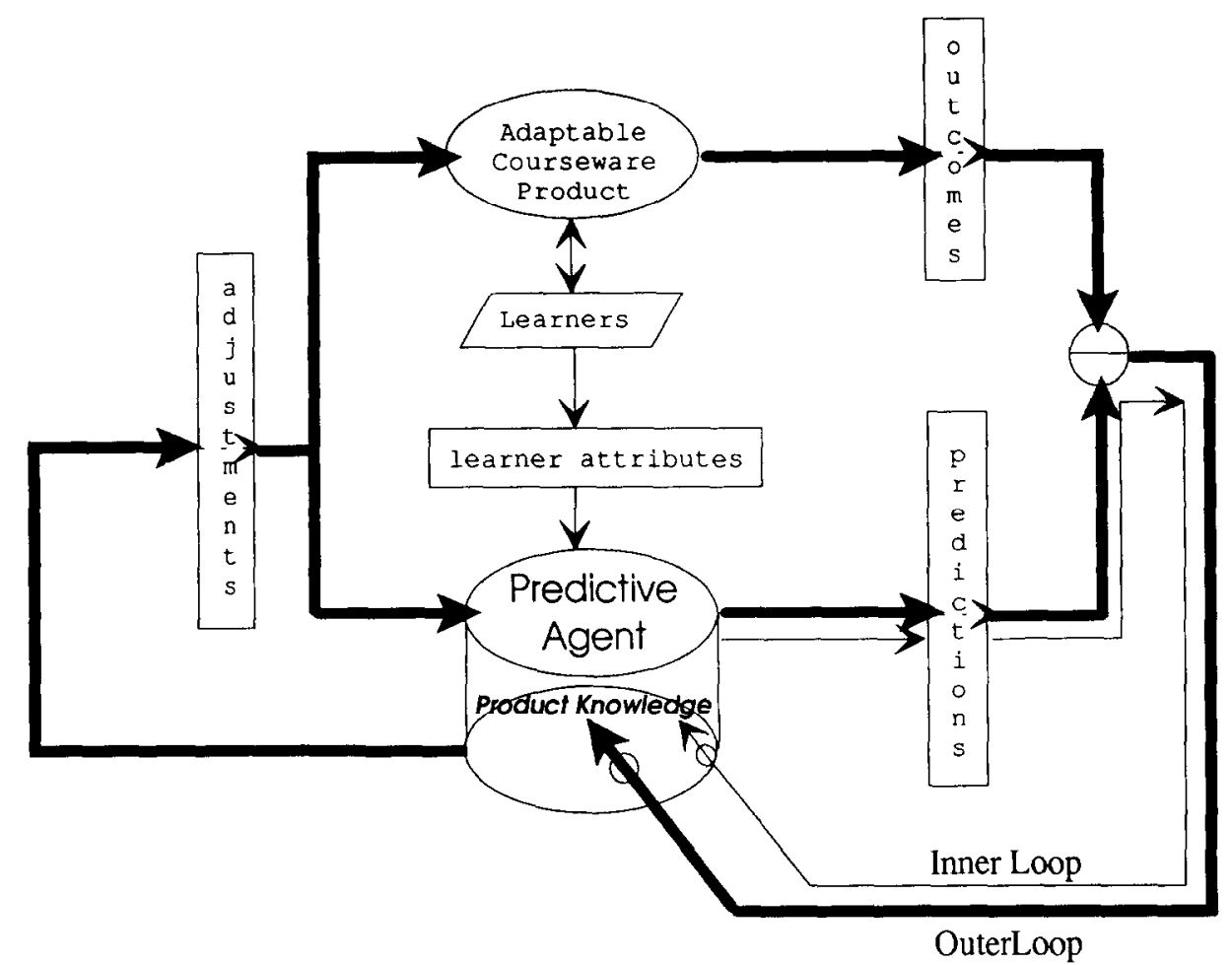

Figure 4. A framework for inductive acquisition of product knowledge.

\section{Some Methods for Automating the Inner Loop}

Automating the inner loop consists of automating Step 2, determination of the discrepancies between predictions and factual outcomes, and 3, refinement of the knowledge base. Automation of Step 2 consists of the automatic calculation of the error between predicted outcomes and factual outcomes. Presuming that outcomes can be represented as vectors and a formula for calculating the error is available, it is feasible to automate this step. More difficult is the automatic refinement or extension of the knowledge base. We consider the refinement of the knowledge base as a so-called credit or debit assignment problem. Automatic extension of the knowledge base with new relations is considered as a so-called seeding problem.

First the credit or debit assignment problem. Those parts of the knowledge base that contribute to a correct prediction are to be credited, and parts contributing to an incorrect solution are to be debited. The assignment problem can be notably complicated by considering situations in which many relations in a knowledge base are involved in generating an output result. The relations may be coupled, in which case internal results from specific relations are passed to other relations, causing a chain of relations involved in producing an output result. One way to assign credit or debit is by back-propagating errors in the output result through the chain of relations, starting at the end of the chain and finishing at the beginning of the chain. The back-propagated error information is used to punish or reward relations in the decision chain, according to their contribution to the error made. Freeman and Skapura (1991) and Holland (1986) provide several algorithms for the automatic assignment of credit or debit. 
The more difficult seeding problem consists of extending the knowledge base with new relations. Once these new relations are generated, they can be reinforced or weakened by credit or debit assignment. Genetic algorithms exploit subtle random methods to create "offspring" relations that are plausible to be successful in surviving the credit or debit assignment competition with already existing relations (Goldberg, 1989; Holland et al., 1986). Artificial neural networks (Simpson, 1990) apply a different strategy. They exhibit a redundancy in the knowledge base by using latent relations. These latent relations have zero or very small strength, and are therefore not involved in the reasoning process. In the process of learning, the zero-strength relations may become active by revisions of the strength due to a credit assignment. By using neural networks or genetic algorithms, it is possible to automate the creation of new relations.

\section{Some Methods for Automating the Outer Loop}

Automating the outer loop consists of automating Step 1, generating new adjustments, and Step 2, determining factual outcomes. Automation of Step 3, expanding the knowledge base, is discussed in the previous section. Step 2 may be automated by exploiting integrated software environments for adapting, using, and evaluating products. As the opportunity exists to exploit classroom use of adapted products, complete automation of Step 2 might not be desirable.

Considerably more difficult is the automation of Step 1 . This problem resembles the seeding problem described earlier. Instead of creating new relations, new adjustments have to be generated. Methods usable for automating the seeding problem can be applied for the generation of new adjustments. Given the hyperspace representation, we can consider two additional methods for generating new adjustments. First, regions in the hyperspace that are considerable in size might indicate a lack of specialization. By (random) sampling points within the large region, information may be obtained about the necessity to split the region into subregions. Second, the existence of many small neighboring regions may indicate too much specialization, or may even indicate incorrect values attached to some regions. In this case, (random) sampling points across the adjacent regions may provide information about the possibilities to integrate adjacent regions into larger regions, enabling generalization. The first method tries to reduce the size of the regions, enabling specialization, while the second method tries to enlarge the size of the regions, enabling generalization. Both methods may be used together to try to find an equilibrium between specialization and generalization.

\section{Using the Inductive Knowledge Acquisition Method}

An interesting discussion in respect to the methodology and the framework for inductive knowledge acquisition pertains to the role of the second author in this framework. Three scenarios are possible. The first scenario consists of a situation in which the second author is not involved in knowledge acquisition. The second author only applies the acquired knowledge. The predictive agent is provided to the second author by a first author. For the first scenario, it is preferred to automate both the outer and the inner loop in order to insure efficiency.

In the second scenario, it is assumed that a second author creates a knowledge base by actually using an adaptable courseware product in a classroom situation. Complete automation of the outer loop is not possible in this case because this would eliminate the second author's freedom to adapt the courseware product to personal needs and tastes. A second author is not likely to use such an inflexible 
system. Automation of the inner loop, on the other hand, will not restrict the second author's freedom in the adaptation of a courseware product. An automated inner loop allows for the automatic integration into the knowledge base of the adjustment-outcomes patterns acquired while using an adapted courseware product.

In the third scenario, finally, it is assumed that first an initial knowledge base is created by a first author, and afterwards this knowledge base is refined through use by a second author. Our research concentrates on the second phase of this scenario, where the knowledge base is refined through use by a second author. We refer to this phase as the "use" phase. In the next section we will consider two strategies that can be applied in the use phase to refine and expand an existing knowledge base.

\section{Two Strategies for Refining and Expanding the Knowledge Base in the Use Phase}

The first strategy is a rather passive one. It just consists of monitoring the outcomes of an adapted courseware product in relation to the adjustments made, and using the collected adjustment-outcome pairs to refine and expand the knowledge base. The strategy is based on the hope that eventually the process of continuous revising of an adaptable courseware product by a second author will result in sufficient knowledge for a predictive agent to be of practical use. The strategy does not seem very realistic though, due to lack of direction in the development of the knowledge base. It is unlikely that employment of the passive strategy in real life will lead to a knowledge base that holds usable knowledge.

A more attractive strategy actively involves the second author. We can expect that a second author, working with a predictive agent, is very much interested in finding adjustments that are predicted to have high-grade learning outcomes. The adjustment of adaptable courseware products and, indirectly, the building of the knowledge base can be guided by providing a second author with the possibility of obtaining "preliminary" predictions. These are predictions from the predictive agent that are accompanied by certainty factors, indicating the reliability of a prediction. A certainty factor for prediction may be calculated on the basis of a number of criteria that directly or indirectly provide information about the reliability of predictions. An example in the hyperspace representation of such a criterion may be the distance in the hyperspace between the current point and points that have established high certainty factors. The second author is supposed to use the predictive agent to find an adjustment. Making the certainty factors explicit to the second author could help in deciding whether or not to follow a prediction. In case of a prediction with low certainty, the second author is supposed to make an adjustment according to his own judgement of the situation. A possible guideline for the second author concerning how to use the predictive agent in order to find suitable adjustments may consist of the following steps:

1a. Let the second author use a predictive agent to find a collection of adjustments that are predicted to have better learning outcomes than the currently achieved learning outcomes.

1b. If the collection is empty then continue with $4 \mathrm{e}$.

2a. Let the second author selcct from this collection of possible adjustment those that are in agreement with personal tastes and needs.

$2 \mathrm{~b}$. If the remaining collection is empty then continue with $4 \mathrm{e}$.

3a. From the resulting collection, let the second author select those adjustments that are predicted with a high certainty factor. 
3b. If such adjustments can not be found then continue with $4 a$.

$3 \mathrm{c}$. Adjust the courseware product according to the adjustments found and continue with 5 .

4a. From the resulting collection, let the second author select those adjustments that are predicted with a medium certainty factor.

$4 \mathrm{~b}$. If such adjustments can not be found then continue with $4 \mathrm{e}$.

$4 \mathrm{c}$. Let the second author try to predict the effects of the selected adjustments.

4d. If the second author is able to find in the resulting collection of adjustments such adjustments that are considered to be good ones, then continue with $3 \mathrm{c}$.

$4 \mathrm{e}$. Let the second author make adjustments on the basis of his or her own judgments.

5. Let the second author use the courseware product in a classroom situation.

6 . Let the second author evaluate the product and determine the learning outcomes.

7. If the second author thinks that the product still needs to be improved then continue with 1.

8. End.

It is necessary to develop tools for Steps 1a, 2a, 3a, 4a, and 6 in order to simplify the selection task.

The iterative process of the second author making an adjustment on basis of preliminary predictions, evaluating the courseware product, and refining the product should converge to an adjusted product that can be associated with high-grade learning outcomes. A problem in this process is that, in spite of better solutions, it may converge to a product that embodies a suboptimal solution. Such a suboptimal solution can be conceived of as a local maximum learning outcome in the hyperspace. In such a case, the not fully predictable (human) behavior of the second author can be beneficial. As a result of the human decision "noise" introduced by the second author, the current solution may hop out of the pitfall of the local maximum, hopefully on its way to the global maximum. Techniques to escape local maxima are described by Freeman and Skapura (1991), Simpson (1990), and Zeidenberg (1990).

While a second author is refining a product, in the background a process is running that automatically updates a product knowledge base. When a second author finishes refining an adaptable courseware product, the knowledge base can be stored for future reuse and refinement. In the next section, we will consider artificial neural networks as a technique that may be used to automate the inner loop.

\section{NEURAL NETWORKS AS A MEANS FOR IMPLEMENTING THE AUTOMATION OF THE INNER LOOP}

Neural networks are mathematical models of theorized mind and brain activity that exploit the massively parallel local processing and distributed representations that are believed to exist in the brain. According to Zeidenberg (1990), the role of a neural network is to perform a function that associates each input pattern with an output pattern. Neural network learning algorithms, such as back-propagation (Freeman \& Skapura, 1991), use the statistical properties of a set of input-output pairs, called the training set, to generalize input-output relations. An important advantage of neural networks is that they allow for exceptions and randomness in 
the association between paired variables. Traditional rule-based systems are based on deduction, and are therefore deterministic. Where rule-based systems in many cases will not provide a conclusion, neural networks will always give an output. In case of a novel situation, the output of a neural network is a generalization on basis of the learned knowledge. Also, neural networks are flexible and train on numerical data by example. It is not a simple task to modify a large symbolic knowledge base with many hundreds of rules. A third advantage of neural networks is that generating an output generally does not require much processing. An output can be generated almost instantly. An important disadvantage of neural networks is that finding a correct network configuration and successful learning rules is a difficult task.

It is feasible to implement a predictive agent as an artificial neural network. The representation of the adjustments has to be transformed to a representation that is suitable for use as an input representation for a neural network. The output of a neural network has to be transformed in such a way that it can be interpreted as a specific learning outcome. For using a predictive agent to provide preliminary predictions, it is necessary to expand the output of a neural network with certainty factors on basis of the hyperspace of this neural network and its learning history. This is an interesting research question to solve.

Examples of the use of neural networks to classify human behavior are rare. Beale and Finlay (1989) have used a neural network to classify computer users into various categories based upon users' command responses in an exploratory functional programming environment. After learning by example, their system, in four tests, was able to distinguish experts from novices with approximately $70 \%$ accuracy. Mengel (1990) used synthetic data representing simulated student behavior while working on subtraction problems to train neural networks in order to be able to predict student behavior in intelligent tutoring systems. She concluded that neural networks have far more predictive power than other student modelling methods in the presence of incomplete information. Mengel and Lively (1991) try to encourage the use of neural networks in intelligent educational systems: "Considering neural networks now will allow the designer to make better use of networks in intelligent tutoring systems as new rescarch brings more information about the characteristics of neural networks to light" (p. 55).

\section{CONCLUSIONS}

Adaptable courseware products should offer their users, mainly learners and teachers (more generally called second authors), a fair degree of freedom in modifying products to their own needs and tastes. With the increased freedom of the users, however, the complexity of courseware modification becomes manifest. Cognitive complexity stems from the fact that even within a single product a very large number of modification options exist, yet it is unclear what learning outcomes could result from the various modifications. Our intention is to offer second authors decision support in product adaptation. The type of decision support facility that we favor has been termed predictive agent, as its main function is to predict likely learning outcomes from intended adaptations.

The basic problem opposing the effort to realize such a predictive agent is the lack of knowledge upon which the agent could make its predictions. In fact, what knowledge is involved? And how could we obtain it? According to our opinion, 
three "types" of knowledge come into play during the process of product adaptation: product, process, and tool knowledge. Our research is focused on product knowledge, as the availability of this type of knowledge should be considered paramount for guiding product modifications. Product knowledge in our admittedly limited view pertains to relations between product attributes, learner attributes, and learning outcomes.

Procuring such knowledge is hard. Interviewing "experts" will not result in a satisfactory amount of this type of knowledge with the desired degree of explicitness, nor can present-day instructional design theories and models offer such explicitness. We have to take refuge, therefore, in the empirical approach. In this article, we have proposed a methodology for knowledge acquisition. This methodology utilizes methods and techniques from the field of machine learning to support automatic knowledge acquisition. The methodology for inductive knowledge acquisition is described by us in a conceptual framework. The framework is to be enriched with methods and techniques enabling efficient and effective acquisition of product knowledge. We have given suggestions to incorporate the second author as participant in the knowledge acquisition process. While the second author is involved in adjusting a product, a knowledge base is incrementally formed in the background. When a second author has finished refining a product, the knowledge base can be stored for future reuse and refinement.

The presented framework offers guidelines for additional research. The following research problems have to be solved:

1. The methodology is embodied in a number of procedural steps. These steps need to be worked out.

2. We have presented guidelines to involve a second author in knowledge acquisition. These guidelines need empirical verification.

3. Methods and techniques to automate knowledge acquisition have been discussed. If these prove not to be suitable for our purposes, additional ones have to be developed.

4. The methodology for inductive knowledge acquisition is based on adapting, using, evaluating, and refining adaptable courseware products. We need to design and implement a prototype of such a courseware product, linked with the framework for knowledge acquisition.

In the title of this article, the striving for the acquisition of instructional design knowledge is made manifest with the phrase "knowledge for courseware engineering." We have proposed the development of a reflective, self-learning system in order to obtain this type of knowledge. We are convinced that such an inductive approach will ultimately offer a significant contribution to courseware engineering activities aimed at high productivity and sound product quality.

\section{REFERENCES}

Beale, R., \& Finlay, J. (1989). User modelling with neural systems (Tech. Rep. No. YCS118). Heslington, England: University of York, Department of Computer Science.

Boose, J. H. (1989). A survey of knowledge acquisition techniques and tools. Knowledge Acquisition, 1, 3-37.

De Diana, I. P. F., \& de Vries, S. A. (1990). Adaptable educational courseware: An antidote to several portability problems. Journal of Research on Computing in Education, 23, 225-242. 
Freeman, J. A., \& Skapura, D. M. (1991). Neural networks, algorithms, applications and programming techniques. New York: Addison-Wesley.

Goldberg, D. E. (1989). Genetic algorithms in search, optimization and machine learning. New York: Addison-Wesley.

Holland, J. H. (1986). Escaping brittleness: The possibilities of general-purpose learning algorithms applied to parallel rule-based systems. In R. S. Michalski, J. G. Carbonell, \& T. M. Mitchell (Eds.), Machine learning: An artificial intelligence approach: Vol. 2 (pp. 593-623). Los Altos, CA: Kaufmann.

Holland, J. H., Holyoak, K. J., Nisbett, R. E., \& Thagard, P. R. (1986). Induction: Processes of inference, learning and discovery. London: MIT Press.

Ladhani, A. N., \& De Diana, I. P. F. (in press). Computer aided courseware engineering and performance modelling tools. In I. P. F. De Diana \& P. van Schaik (Eds.), Lecture notes in computer science: Aspects of courseware engineering. Twente, The Netherlands: University of Twente.

Li, Z., \& Merrill, M. D. (1990). Transaction shells: A new approach to courseware authoring. Journal of Research on Computing in Education, 23, 72-86.

Mengel, S. (1990). Using neural networks to predict student behavior in intelligent tutoring systems. Unpublished doctoral dissertation, Texas A\&M University, College Station, TX.

Mengel, S., \& Lively, W. (1991). On the use of neural networks in intelligent tutoring systems. Journal of Artificial intelligence in Education, 2(2), 43-56.

Merrill, M. D. (1983). Component display theory. In C. M. Reigeluth (Ed.), Instructional design theories and models: An overview of their current status (pp, 279-335). Hillsdale, NJ: Erlbaum.

Merrill, M. D. (1987). The new component display theory: Instructional design for courseware authoring. Instructional Science, 16, 1-10.

Merrill, M. D., \& Li, Z. (1989). An instructional design expert system. Journal of Computer-Based Instruction, 16, 95-101.

Merrill, M. D., Li, Z., \& Jones, M. K. (1990). Second generation instructional design (ID2). Educational Technology, 30(2), 26-31.

Moonen, J. (1989). Courseware development at the crossroads?. Education and Computing, 5, 103-109.

Muggleton, S. (1990). Inductive acquisition of expert knowledge. Glasgow, Scotland: Turing Institute Press.

Salter, W. J. (1988). Human factors in knowledge acquisition. In M. Helander (Ed.), Handbook of human-computer interaction (pp. 957-968). Amsterdam: Elsevier.

Simpson, P. K. (1990). Artificial neural systems: Foundations, paradigms, applications, and implementations. Elmsford, NY: Pergamon.

Wenger, E. (1987). Artificial intelligence and tutoring systems. Los Altos, CA: Kaufmann.

Woods, D. D., \& Roth, E. M. (1988). Cognitive system engineering. In M. Helander (Ed.), Handbook of human-computer interaction (pp. 3-43). Amsterdam: Elsevier.

Zachary, W. (1988). Decision support systems: Designing to extend the cognitive limits. In M. Helander (Ed.), Handbook of human-computer interaction (pp. 997-1031). Amsterdam: Elsevier.

Zeidenberg, M. (1990). Neural network models in artificial intelligence. New York: Ellis Horwood. 